\title{
A FRAMEWORK FOR A SMARPHONE NATURAL LANGUAGE- DRIVEN HEALTHCARE MONITOR AND ADVISOR USING WEARABLE DEVICES
}

\author{
George Stefanek, Purdue University Northwest, stefanek@pnw.edu \\ Marianne Curia, Purdue University Northwest, mcuria@pnw.edu \\ Janet Garwood, Purdue University Northwest, jgarwo07@pnw.edu
}

\begin{abstract}
The use of wearable technologies to monitor various health and fitness parameters are becoming wide spread through the use of fitness trackers and other wireless monitoring devices. These relatively inexpensive wearable technologies can now be practically brought together to provide continuous monitoring of a patient at home and gather additional health-related data by using natural language processing (NLP) to interact hands-free with the patient. The health parameters gathered though these mechanisms can provide a snapshot of the patient's state of health and can be processed through a rule-base to provide alerts and advice. Such a system makes it easier for health compromised and older populations of patients to communicate with the system and be monitored seamlessly through wearable devices. A framework for such a system is proposed and applied to the domain of Congestive Heart Failure (CHF).
\end{abstract}

Keywords: Healthcare Information Systems, Wearable Technologies, NLP, Healthcare Smartphone Applications

\section{INTRODUCTION}

Natural language processing (NLP) technologies are increasing in use today and include web-based systems such as Amazon's Alexa, Apple's Siri, and Google's Assistant, and embedded NLPs such as Lingpipe. Other technologies in wide-spread use today are wearable devices such as fitness trackers from vendors such as Fitbit that monitor heart rate and various other exercise parameters, wearable pulse oximeters from Withings, Garmin, and others that monitor oxygen saturation, emerging wearable devices such as Omron's HeartGuide, ThinkBand, and WearPai that monitor blood pressure, and AliveCor's KardiaBand that monitors heart rhythm. These relatively inexpensive off-the-shelf technologies can now be brought together to provide continuous monitoring of various health parameters. An architecture for a system is proposed that integrates NLP, wearable devices, a medical rule-base, and alerting subsystem to create an integrated system that is inexpensive and can be used at home in the medical domain. The initial proof-ofconcept system will be applied to the monitoring of Cardiac Heart Failure (CHF) patients at home using commercialoff-the-shelf wearable devices that can be combined with an easy-to-use natural language user-interface for communication between a patient and the smartphone app. Such an integrated system can provide alerts and expert advice, which may lead to improved quality of life and reduced cost to the healthcare industry by the continuous monitoring of a patient and an affordable option for patients.

Such a system goes beyond traditional health monitoring systems that monitor health parameters (Alwan, 2006; Ogawa \& Togawa, 2000; Tamura, Ogawa, Yoda, \& Togawa, 1998), but will integrate multiple technologies that can seamlessly interact with a person by natural language communication and offer alerts and advice in focused medical domains in order to facilitate optimum health and early intervention for emerging health issues. There is documented research that describes smart healthcare system frameworks (Larssan, \& Hayes-Roth, 1998; Demirkan, 2013; Roy, Pallapa, \& Das, 2007; Röcker, 2010; Röcker, \& Maeder, 2011) which are more limited in breadth for integrating health data and providing intelligent feedback. Recent research takes input from diverse modalities, such as sensors, user profile information, social media, clinical knowledge bases, and medical experts to generate standards-based personalized recommendations (Hussain et al., 2013). Apple has also begun a heart study where they explore developing an Apple phone app to identify irregular heart rhythms including those from potentially serious heart conditions such as atrial fibrillation using data from an Apple watch with a built-in electrocardiogram ("Apple Heart Study”, 2019; Keown, 2019; “Apple Heart Study launches to identify irregular heart rhythms", 2019). The app that is 
the subject of this paper is focused on not only integrating information from multiple modalities, but also acquiring additional information directly from a patient through the use of a natural language interface. The interface allows the person to directly converse with the system in a natural way and is deployed on a smartphone that integrates with commercial off-the-shelf wearable device technology.

\section{STATEMENT OF THE PROBLEM}

Congestive heart failure (CHF) (Congestive Heart Failure and Heart Disease, 2018) is a condition in which the heart cannot pump enough blood and nutrients to meet the body's need for oxygen and other needs. The inability to pump enough blood manifests symptoms that include fluid retention that causes edema in the lower extremities and/or abdomen, weight gain, shortness of breath, lack of energy and the loss of quality of life. Patients with CHF often have multiple co-morbidities such as hypertension, diabetes and coronary artery disease that require ongoing monitoring of the patient status. The annual cost of care for CHF patients is $\$ 30.7$ billion and is estimated to increase to $\$ 70$ billion by 2030 (Hale et al., 2016). Heart failure is also one of the most common reasons for hospital admissions for patients over the age of 65 (American Heart Association, 2017).

\section{SIGNIFICANCE OF THE PROBLEM}

In order to monitor patient health at home and improve the state of health of the CHF patient and decrease hospitalizations, a system is proposed that monitors the patients and provides alerts and advice to the patient using technologies that facilitate easier and seamless interaction with older, health compromised patients through the use of natural language communication and the use of wearable technologies on a smartphone platform. Strategies such as improved communication with health care providers, and close monitoring of high-risk populations are needed to achieve lower readmission risk (Ziaeian, \& Fonarow, 2015).

\section{INTELLECTUAL MERIT}

Currently, there is no known smartphone application that seamlessly integrates multiple wearable medical monitoring technologies, together with expert medical knowledge, to assist a person in making intelligent decision regarding their heal using a more natural language-driven method of communication between the patient and the smartphone app.

This type of architecture advances knowledge in the area by integrating natural language processing into the healthcare area directly with patient responses to critical medical questions. It also seamlessly integrates multiple, inexpensive commercial-off-the-shelf wearable and other wireless technologies to provide the healthcare provider in-home monitoring of patients with medical problems using widely available wearable technology integrated with an app that runs on Android and iOS smartphones.

The Smart Health Status Monitor and Advisor architecture combines wearable technology with telemedicine and allows for the collection of individualized data. This data can be utilized to provide patient-centered, personalized care in a cost-efficient manner since the application allows the patient to monitor their status while remaining in their home. By tracking key indicators, the app can allow for early intervention. Prompt intervention and early management of symptoms may possibly reduce hospital readmissions as well as improve the quality of life for the patients diagnosed with heart failure.

\section{METHODS}

The methods used in the design of a system architecture for the integration of technologies for NLP, wearable devices, advice, alerts, and local and remote databases included:

- Identify the health parameters to be collected for the targeted medical domain, 
- Identify the availability of wireless, Bluetooth-compatible wearable devices that can collect data for as many of the identified health parameters for the target application.

- Identify requirements for the wireless, wearable devices such as accuracy, FDA clearance (if needed), availability of APIs, Bluetooth compatibility.

- Identify user experience (UX) and user interface (UI) requirements.

- Identify internet connectivity requirements (internet readily available or not).

- Identify natural language processing technology to be used and the vocabulary and phrases that are required to understand communication in the health-related area for which the application is focused (in the prototype example the target medical area was Cardiac Heart Failure).

- Identify the operating systems and minimum version of the smartphone OS for the target application.

- Identify subsystem interface requirements and functionality.

- Identify phone hardware requirements.

- Identify the development technologies.

In this proof of concept prototype app the target platform was Android version 6 or lower. A Fitbit Aria scale, Fitbit Surge watch, and Lingpipe NLP were initially used in the proof-of-concept system. Development was done using Xamarin (a mobile applications cross-platform development environment integrated into Microsoft's Visual Studio IDE) and Java using multiple student teams working on various modules of the application of which portions where implemented on the smartphone platform and other modules implemented as a Java client-server application. All modules are now being integrated into a Smartphone application using Xamarin. The prototype app focuses on applying this technology to the CHF domain and currently has implemented parts of the proposed architecture including an interface to a Fitbit cloud database for downloading Fitbit smartwatch data from the cloud to the app, a mobile graphical user-interface that allows a patient to answer questions posed by the app regarding a patient's health status, a rule-base that provides advise based on the Fitbit data and patient responses to the app questionnaire, and an Alerter module that issues alerts to the patient based on patient status. Additionally, use of the LingPipe NLP has been tested in a separate app that gathers general medical history data. The NLP was trained only on family history terminology and phrases and has not yet been integrated into this app and trained on the vocabulary and phrases needed to respond to the app's questionnaire related to CHF.

\section{DESIGN}

The key features for this app are: 1) monitor a person's health parameters by using wearable or wireless commercialoff-the-shelf devices such as Fitbit, 2) perform interviews with a person to retrieve health related information using natural language communication, 3) provide health-related advice through the use of a rule-base by examining the state of all healthcare parameters for a patient which are monitored and updated periodically, 4) provide alerts and warnings to the patient and healthcare providers, and 5) communicate the advice via synthesized speech and display it on a smartphone user-interface.

This architecture can be used to develop applications that could be deployed on a smartphone or tablet as an Android, iOS or Universal Windows application and interface with a variety of wearable and other wirelessly connected health monitoring devices that collect health parameter data.

The health parameters that were targeted to be gathered from wearable devices for the proof-of-concept app where related to monitoring CHF patients and included: 1) heart rate, 2) oxygen saturation, 3) exercise (steps walked, stairs climbed), 4) weight, and 5) blood pressure. Additional health-related information not obtainable through wirelessly connected devices, such as: 1) intake and output of fluid, 2) shortness of breath, 3) anxiety level, 4) quality of life / energy level, edema, and 5) activity tolerance would be collected through patient interviews that would be conducted by the app using natural language interaction. Figure 1 shows the architecture of the system with modules for the mobile device user-interface, speech recognition, NLP interface, interview, alerts, advising, rule-base processing, logging, mail, speech synthesis, wearable device interaction, external DB syncing, and local database interaction. Each module consists of a set of classes that can be called from other modules. 


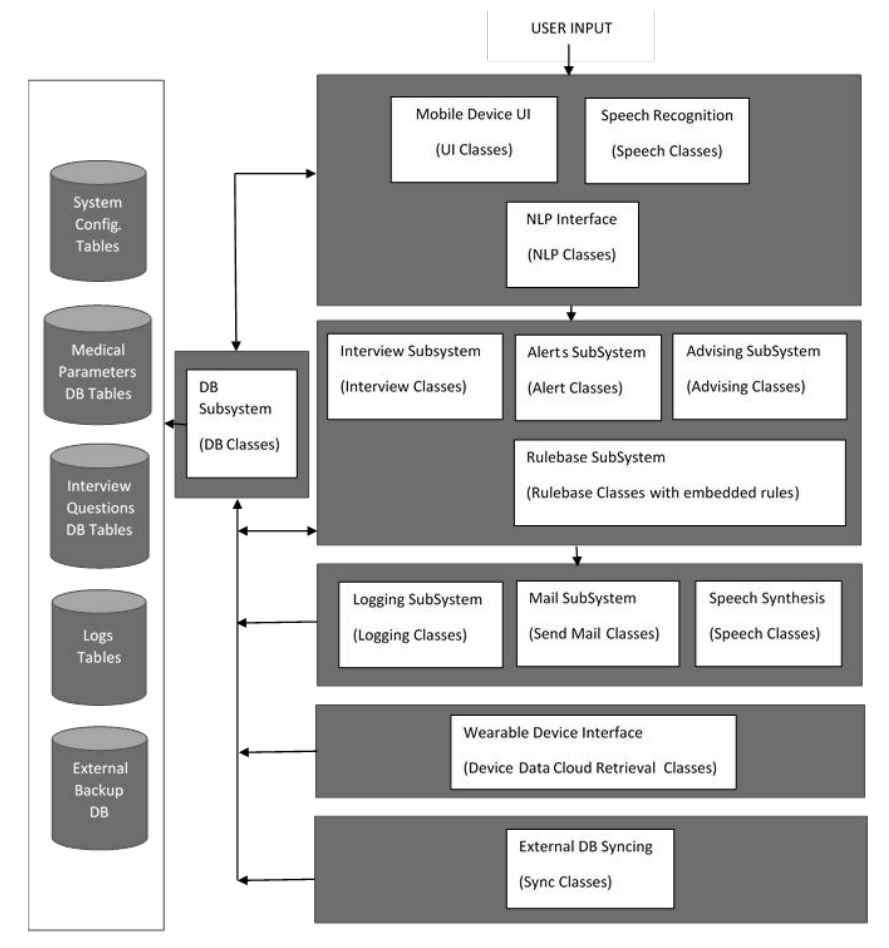

Figure 1. App Architecture.

\section{TECHNOLOGIES}

The destination hardware for this app are Android and iOS smartphones and tablets and Universal Windows tablets. The app will use a variety of wearable and wireless technologies that monitor a variety of health parameters and store data collected from the wearable and/or wireless devices on the various vendor cloud databases. The application will retrieve the cloud-based data using a vendor's API to access their cloud databases and copy and store the data locally in the smartphone's SQLite database. The devices will include: 1) a Fitbit fitness tracker watch that monitors heart rate and a patient's exercise via steps walked and stairs climbed, 2) a Fitbit wireless scale that measures weight and BMI, 3) wearable pulse oximeters from Withings or Garmin or other manufacturers that monitor oxygen saturation, 4) a wearable device that monitors blood pressure such as upcoming Omron's HeartGuide or ThinkBand or WearPai. These technologies will require the vendor to have an API which can be used to get access to the data gathered by the devices. Existing, as well as new and emerging technologies which are FDA cleared will be evaluated. If a wearable device is not available, then a wireless, but non-wearable device may be substituted.

The application gets the state of the patient by periodically retrieving the health parameter data from the local smartphone SQLite database and loading it into a patient state object in the app. The patient state health parameters are compared against the rule-base in the application in order to issue warnings, alerts or advice.

The health parameters that cannot be monitored using a wearable or wireless device such as intake and output of fluid, shortness of breath, anxiety level, quality of life / energy level, edema, and activity tolerance will be collected via the application's automated interview with the patient. The automated interview is implemented as a decision tree that drives the question asking process. The decision tree nodes will be stored as records in a relational database table in the smartphone's local SQLite database, and an interview program queries the database to ask the appropriate questions. The questions are displayed on the screen and will also be synthesized as speech. The patient responds to the questions via voice input or by interacting with the smartphone user-interface. Currently, in the prototype system, technologies such as Dragon voice recognition software have been used for this purpose. 
The translated speech to text will be processed by a natural language processor (NLP) that will attempt to understand the patient's response to a question. The NLP software will be trained extensively with training sets of various medical terminology and lists of potential patient responses to every question. Currently, the prototype system uses an integrated natural language processor toolkit called LingPipe from Alias-i. This NLP can be embedded in the application and does not require an internet connection to a cloud-based NLP service. Additional NLP APIs such as Google's Cloud Speech API and Amazon's API for natural language processing will be explored as an alternative to an embedded NLP in the application.

The rule-base to make intelligent decisions and provide alerts and warnings is implemented as custom code in C\# within the application. Alternatively, a rule-base engine can be used as the rule-base becomes more complex or requires an inference engine to be processed. The use of a rule-base engine such as DTRules has been explored in the project, but currently is not required because of the limited complexity of the rule-base. Also, an Alerter subsystem will cycle at a to-be-determined rate to capture a snapshot of the health parameters from the local database and compare the state of the health parameters against the rule-base. Any rules that are triggered will provide an alert or recommendation.

The system will be implemented as a mobile application using Xamarin in Visual Studio for cross-platform development of mobile applications. The Xamarin platform allows for the coding of an application using the C\# language and creates target applications for Android, iOS, and Universal Windows without having to write specific versions of the code in Java for Android or Swift for iOS. The code will be written using C\# in Xamarin and will be implemented as a Xamarin forms cross platform application.

The application will store a patient's health parameter data and other logs of recommendations and interactions with the patient in the local database. Any flat files will be encrypted using 256-bit AES encryption. Technologies to encrypt the SQLite database will be explored such as SQLCipher - an extension in SQLite that provides 256-bit AES encryption of database files and a technique and/or product chosen.

Reports will be written out as flat files, converted to PDF format and encrypted using 256-bit encryption. Encrypted reports generated to a healthcare provider will be sent to the healthcare provider via an email interface in the application.

Wearable technologies from Fitbit that monitor heart rate and exercise have been integrated into the prototype system. Also, weight and body mass index (BMI) are collected via Fitbit's Aria wireless scale. A vendor(s) that provides a wearable wireless device for monitoring blood pressure and oxygen saturation will be selected from the vendors that provide FDA cleared devices.

\section{CONCLUSIONS}

The architecture provided here can support development of interfaces to any number of other wearable or wireless devices, any NLPs either embedded or web-based that provide APIs, and creation of rule-bases that can be customized to focus on specific processing of health parameters for specific medical problems. NLPs can be trained with training sets of terminology and phraseology for specific medical areas to which the system is focused. As new NLP technologies and wearable technologies area made available wrapper classes can be written to use the new technologies. Currently, a Xamarin app collects data from cloud-based Fitbit databases and stores the data on the local database and displays the state of the patient. The prototype system was initially implemented in Java as a clientserver application and currently, pieces such as the Alerter module, interview modules, and mobile user-interface are being moved to the mobile platform using Xamarin and C\#. The author will continue development of the system targeted for the CHF medical area until the system is complete, tested and its efficacy can be tested on an initial patient population of 20 patients. 


\section{REFERENCES}

Alwan, M., Mack, D. C., Dalal, S., Kell, S., Turner, B., \& Felder, R. A. (2006, April). Impact of passive in-home health status monitoring technology in home health: Outcome pilot. In Distributed Diagnosis and Home Healthcare, 2006. D2H2. 1st Transdisciplinary Conference on (pp. 79-82). IEEE.

American Heart Association. (2017, May). Causes and risk factors of heart failure. Retrieved August 21, 2017 from www.heart.org

Apple Heart Study. (2019). Retrieved July 11, 2019 from http://med.stanford.edu/appleheartstudy.html

Apple Heart Study launches to identify irregular heart rhythms. (2019). In Apple Newsroom online. Retrieved July 11, 2019 from https://www.apple.com/newsroom/2017/11/apple-heart-study-launches-to-identify-irregularheart-rhythms/

Congestive Heart Failure and Heart Disease. (2018). Retrieved from https://www.webmd.com/heart-disease/guideheart-failure\#1

Demirkan, H. (2013). A smart healthcare systems framework. IT Professional, 15(5), 38-45.

Hale, T. Jethwani, K., Singh Kandola, M., Saldana, F., \& Kvedar, J. (2016, May). A remote medication monitoring system for chronic heart failure patients to reduce readmissions: A two arm randomized pilot study. Journal of Medical Internet Research. doi:10.2196/jmir.5256

Hussain, M., Khattak, A. M., Khan, W. A., Fatima, I., Amin, M. B., Pervez, Z., ... \& Saddiqi, M. H. (2013). Cloudbased Smart CDSS for chronic diseases. Health and Technology, 3(2), 153-175.

Keown, A. (2019). J\&J and Apple Team up for AFib Program With Apple Watch. Retrieved July 11, 2019 from https://www.biospace.com/article/j-and-j-and-apple-team-up-for-heart-health-program/

Larssan, J. E., \& Hayes-Roth, B. (1998). Guardian: intelligent autonomous agent for medical monitoring and diagnosis. IEEE Intelligent Systems and their Applications, 13(1), 58-64.

Ogawa, R., \& Togawa, T. (2000). Attempts at monitoring health status in the home. In Microtechnologies in Medicine and Biology. 1st Annual International, Conference On. 2000 (pp. 552-556). IEEE..

Röcker, C. (Ed.). (2010). Smart Healthcare Applications and Services: Developments and Practices: Developments and Practices. IGI Global.

Röcker, C., \& Maeder, A. (2011). User-centered design of smart healthcare applications. Electronic Journal of Health Informatics, 6(2), 8-14.

Roy, N., Pallapa, G., \& Das, S. K. (2007, October). A middleware framework for ambiguous context mediation in smart healthcare application. In Wireless and Mobile Computing, Networking and Communications, 2007. WiMOB 2007. Third IEEE International Conference on (pp. 72-72). IEEE.

Tamura, T., Ogawa, M., Yoda, M., \& Togawa, T. (1998). Fully automated health monitoring system in the home. IEEE Transactions on Electronics, Information and Systems, 118(7-8), 993-998.

Ziaeian, B., \& Fonarow, G. C. (2015). The Prevention of Hospital Readmissions in Heart Failure. Progress in cardiovascular diseases, 58(4), 379-85. 